hep-th/9412298

\title{
Dill: An Algorithm and a Symbolic Software Package for Doing Classical Supersymmetry Calculations
}

\author{
Vladan Lučić \\ Physics Department \\ Northeastern University, Boston, MA 02115, USA \\ e-mail:vlucic@lynx.neu.edu
}

\begin{abstract}
An algorithm is presented that formalizes different steps in a classical Supersymmetric (SUSY) calculation. Based on the algorithm Dill, a symbolic software package, that can perform the calculations, is developed in the Mathematica programming language. While the algorithm is quite general, the package is created for the $4-D, N=1$ model. Nevertheless, with little modification, the package could be used for other SUSY models. The package has been tested and some of the results are presented.
\end{abstract}

\footnotetext{
${ }^{1}$ The work was supported in part by DOE grant no. DE-FG02-85ER40233
} 


\section{Introduction}

SUSY and supergravity theories have been candidates for unifying electro-weak, strong and possibly gravitational interactions [1]. They are also used as effective theories, describing the low-energy behavior of a more fundamental theory, as is the case with the string theories [2]. Many calculations in such theories, on the classical level, include tedious but conceptually straightforward manipulations. A typical object that is manipulated is an expression that contains both commuting and anticommuting spinor and tensor fields as well as superfields with different indices. Typically, one has space-time, spinor and indices denoting extended representations (for $N>1$ ). Furthermore, a field may have a definite symmetry with respect to the interchange of some of its indices. Also, since at least some of the fields are anticommuting, there are nontrivial symmetries with respect to interchange of the order of the fields. All this makes it more difficult to keep track of signs, to recognize and group or cancel the same terms, or to eliminate the terms that vanish due to the symmetry properties.

There are also operations that are often used, such as the application of different kinds of derivatives: ordinary, Grassman, or covariant. Again, the rules are clear, but the actual application can be tedious.

In recent years, symbolic programming packages have been developed for different problems, such as calculation of Clebsh-Gordan and Rachah coefficients [3], tensor products of Lie algebras [4], in differential geometry [5] [6], general relativity [7] [8], gamma matrices manipulation [9], and for the calculation of Feynman diagrams [10], to mention just a few. When the importance of SUSY was realized, symbolic packages were developed for problems related to it: super-Feynman diagrams [11, Lie superalgebra manipulations [12], calcula- 
tion of the supertrace of a supermatrix [13] and the anomaly calculation in superstring theories [14], among others. Having in mind the manipulations needed in actual SUSY and supergravity calculations, it seemed natural to develop an algorithm and implement it in one of the symbolic programming languages that could perform some of the manipulations, thereby reducing the effort one has to make in performing the calculations. The idea is not new; a package was developed in REDUCE [15] for exterior calculus in superspace and the construction of supergravity actions [16]. In the last few years some very sophisticated and flexible symbolic programming systems were developed (e.g. Mathematica [17] and Maple [18) and used in different symbolic programming projects [5] [9] [10]. An algorithm and a package based on a symbolic programming system, that could perform analytical calculations in SUSY, would make it possible not only to use the package by itself, but also to use the commands of the symbolic system to further process the output of the package, or to integrate the package with some other, giving the user more flexibility in using the package for different problems. Hence, Dill, the software package that can perform classical SUSY calculations, has been developed in Mathematica 2.0. It consists of several separate modules, or functions each doing a specific operation on a given expression. One of the most important functions renames dummy indices and reorders factors in a product in a unique way, so that mathematically equal terms have the same form, therefore making it possible for the underlying symbolic programming system to group the same terms in the expression that is manipulated. The functions can be used separately, or, when a certain sequence of operations is needed, as commands in a program that does the whole sequence. In general, a calculation in SUSY involves many different steps; some of them are technical and straightforward, so they are included in the package, but some require more inventive, 
or "human", manipulations. Therefore, Dill is intended to be used interactively; whenever a user who, while doing a complicated calculation, needs to perform one or more of the calculations defined in the package, he/she simply applies the appropriate function(s), gets a result and continues the calculation on his/her own, using Dill when needed. In order to use the package, one has to have a working knowledge of Mathematica.

An application of a function returns an expression in such a format that another function can be applied on the returned expression. Unfortunately, it is not easy for a user to read such an expression, especially if it is long and has a lot of indices. In order to make it easier, a Mathematica- $\mathrm{T}_{\mathrm{E}} \mathrm{X}$ interface was written in $\mathrm{C}$. It converts the output into a $\mathrm{T}_{\mathrm{E}} \mathrm{X}$ file using conversion rules given by the user in a separate file. The dvi file produced from the $\mathrm{T}_{\mathrm{E}} \mathrm{X}$ file is then shown on a screen using xdvi, or, simply, saved. It is necessary to work under $\mathrm{X}$-Windows and have xdvi program in order to show the file on a screen; otherwise, the dvi file can be simply processed and printed out.

The main body of this article is organized as follows: Section 2 gives a simple overview of SUSY and shows the kind of calculations that can be done on a computer. Section 3 gives the algorithms for specific manipulations and Section 4 explains the way the algorithms are implemented in Mathematica software and gives information about the package for a potential user. Examples are shown in the Appendices.

\section{A Brief Introduction to Supersymmetry Calculations}

The algorithm and the package were developed for use in a SUSY calculations at the classical level. The application on a model other than $4-D, N=1$ requires some definitions 
to be changed and the addition of model-dependent manipulations. Nevertheless, it is general enough to include other calculations involving similar objects and concepts. In this section, after a brief overview of SUSY in general and some additional properties specific to $4-D, N=1$ it is explained what the calculations are, in general, that can be done using Dill.

The usual approach to the SUSY is the superspace formulation (see, for example [19]). Superfields are fields (chiral, vector, ... ) on a superspace which has both ordinary (commuting) space-time coordinates, denoted by $x^{i}$, and Grassman (anticommuting) coordinates, denoted by $\theta^{A, \alpha}$ and the complex conjugate $\bar{\theta}^{A, \dot{\alpha}}$. Here, i is a space-time index, runs from 0 to $D-1$ (where $D$ is the space-time dimension), A from 1 to $\mathrm{N}$ and each of the spinor indices: $\alpha$ and $\dot{\alpha}$, from 1 to the half of the dimension of the spinor representation of the corresponding super-Poincare algebra. (For the dimensions in different models see for example 20].) For $N=1$ SUSY in 4 dimensions, both $\alpha$ and $\dot{\alpha}$ can be 1 or 2 . By convention, a (un)dotted spinor index corresponds to a Grassman coordinate with(out) a bar.

A superfield can be expanded in a Taylor series with respect to the Grassman coordinates. Since the coordinates are anticommuting,

$$
\theta^{\alpha} \theta^{\beta}=-\theta^{\beta} \theta^{\alpha}
$$

it follows that

$$
\theta^{\alpha} \theta^{\alpha}=0 \quad(\text { no summation over } \alpha)
$$

so that Taylor expansion in Grassman coordinates has a finite number of terms. The coefficients in the expansion depend on space-time only, so they are ordinary fields. There are different superfields; for example, the expansion of the chiral superfield $\Phi(x, \theta)$ looks 
like

$$
\begin{gathered}
\Phi(x, \theta)=A+2^{1 / 2} \theta^{\alpha} \psi_{\alpha}+F \theta^{\alpha} \theta_{\alpha}+i\left(\partial_{m} A\right) \theta^{\alpha} \sigma_{\alpha \dot{\alpha}}^{m} \bar{\theta}^{\dot{\alpha}}+ \\
+\left(\partial_{m} \partial_{n} A\right) \eta^{m n} \theta^{\alpha} \theta_{\alpha} \bar{\theta}_{\dot{\alpha}} \bar{\theta}^{\dot{\alpha}} / 4-i \theta^{\alpha} \theta_{\alpha}\left(\partial_{m} \psi^{\beta}\right) \bar{\theta}^{\dot{\beta}} \sigma_{\beta \dot{\beta}}^{m} / 2^{1 / 2},
\end{gathered}
$$

while the vector superfield is

$$
\begin{array}{r}
V(x, \theta)=C+i \theta^{\alpha} \chi_{\alpha}-i \bar{\theta}_{\dot{\alpha}} \chi^{\dot{\alpha}}+i \theta^{\alpha} \theta_{\alpha} \bar{\theta}_{\dot{\alpha}}\left(\bar{\lambda}^{\dot{\alpha}}+i \bar{\theta}^{\dot{\alpha}} \sigma_{\beta \dot{\alpha}}^{m}\left(\partial_{m} \chi^{\beta}\right) / 2\right)+ \\
+i \theta^{\alpha} \theta_{\alpha}(M+i N) / 2-i \bar{\theta}_{\dot{\alpha}} \bar{\theta}^{\dot{\alpha}}(M-i N) / 2-\theta^{\alpha} \sigma_{\alpha \dot{\alpha}}^{m} \bar{\theta}^{\dot{\alpha}} v_{m}- \\
-i \bar{\theta}_{\dot{\alpha}} \bar{\theta}^{\dot{\alpha}} \theta^{\alpha}\left(\lambda^{\alpha}+i \sigma_{\alpha \dot{\beta}}^{m}\left(\partial_{m} \bar{\chi}^{\dot{\beta}}\right) / 2\right)+\theta^{\alpha} \theta_{\alpha} \bar{\theta}_{\dot{\alpha}} \bar{\theta}^{\dot{\alpha}}\left(D+\partial_{m} \partial_{k} C \eta^{m k} / 2\right) / 2,
\end{array}
$$

where summation over the same index, appearing once as a lower and at another place as an upper index, is assumed, as everywhere else in this paper unless otherwise explicitly stated. $A, F, C, M$ and $N$ are commuting, while $\psi, \bar{\psi}, \lambda, \bar{\lambda}, \chi$ and $\bar{\chi}$ are anticommuting fields. By convention the $\sigma$ matrices are given by:

$$
\sigma^{0}=\left(\begin{array}{cc}
-1 & 0 \\
0 & -1
\end{array}\right), \quad \sigma^{1}=\left(\begin{array}{cc}
0 & 1 \\
& \\
1 & 0
\end{array}\right), \quad \sigma^{2}=\left(\begin{array}{cc}
0 & -i \\
i & 0
\end{array}\right), \quad \sigma^{3}=\left(\begin{array}{cc}
1 & 0 \\
0 & -1
\end{array}\right) .
$$

The metric is: $\eta=\{-,+,+,+\}$ and the spinor (Grassman) indices are raised and lowered as

$$
\psi^{\alpha}=\epsilon^{\alpha \beta} \psi_{\beta}, \quad \psi_{\alpha}=\epsilon_{\alpha \beta} \psi^{\beta},
$$

where $\epsilon_{21}=\epsilon^{12}=1, \epsilon^{21}=\epsilon_{12}=-1, \epsilon_{11}=\epsilon^{2}=0$. The convention used here is the one used in [19]; different authors may use other conventions.

Another important concept is that of a Grassman derivative which is also anticommutative, and defined as follows:

$$
\frac{\partial}{\partial \theta^{\alpha}} \theta^{\beta}=\delta_{\alpha}^{\beta}, \quad \frac{\partial}{\partial \theta^{\alpha}} \theta^{\beta} \theta^{\gamma}=\left(\frac{\partial}{\partial \theta^{\alpha}} \theta^{\beta}\right) \theta^{\gamma}-\theta^{\beta}\left(\frac{\partial}{\partial \theta^{\alpha}} \theta^{\gamma}\right)
$$


Finally, the covariant derivatives are:

$$
\begin{gathered}
D_{\alpha}=\frac{\partial}{\partial \theta^{\alpha}}+i \sigma_{\alpha \dot{\alpha}}^{n} \bar{\theta}^{\dot{\alpha}} \frac{\partial}{\partial x^{n}} \\
\bar{D}_{\dot{\alpha}}=-\frac{\partial}{\partial \bar{\theta}^{\dot{\alpha}}}-i \theta^{\alpha} \sigma_{\alpha \dot{\alpha}}^{n} \frac{\partial}{\partial x^{n}}
\end{gathered}
$$

It is necessary to see what kind of calculations are needed in a SUSY theory and what the basic steps needed in such a calculation are. Typically, the calculation involve an application of the covariant derivative on a superfield, or multiplication of two or more superfields and sorting out the result. For the software that can reproduce such a calculation to be developed, the steps have to be clearly identified and put in an algorithmic form. Except when noted otherwise, the steps that follow in this section are used not only in $4-D, N=1$, but in other SUSY theories also. Furthermore, the similar, if not the same, steps are used in any other calculation where both commuting and anticommuting tensor fields are present, so that the algorithm and the package, with possibly minor modifications, can be applied to many different calculations.

The important point to keep in mind is that if a package of this kind is to be written in a language of a symbolic programming system, the steps that the system does and does not do by itself (without additional programs) have to be identified. The latter are the steps that have to be programmed in the package. For example, what the system does by itself is:

$$
a \theta^{\alpha}+b \theta^{\alpha}=(a+b) \theta^{\alpha}
$$

On the other hand, here are few very trivial steps that the system does not do, so a procedure has to be written for each of them:

$$
A \psi^{\alpha}=\psi^{\alpha} A, \quad \psi^{\alpha} \theta^{\beta}=-\theta^{\beta} \psi^{\alpha}, \quad \theta^{\alpha} \theta^{\alpha}=0,
$$




$$
\theta^{\alpha} \theta^{\beta} \theta^{\gamma}=0 \quad(\text { for } 4-D, N=1 \text { only), }
$$

where $A$ is a commuting field and $\psi$ and $\theta$ are anticommuting fields. The multiplication has to be defined as noncommutative. Otherwise, the system would interchange anticommuting fields in a product without putting the necessary minus sign. The widely used summation convention introduces a trivial step from a human point of view:

$$
\theta^{\alpha} \psi_{\alpha}=\theta^{\beta} \psi_{\beta}
$$

In other words, it is not important what the actual symbol for a summation (dummy) index is. Nevertheless, the step requires a complicated procedure if it is to be implemented in a program.

Another set of rules comes from possible symmetries with respect to interchange of two or more indices. If $R_{\alpha, \beta}$ is symmetric and $B_{\alpha, \beta}$ is antisymmetric with respect to interchange of the indices, apart from trivial identities:

$$
R_{\alpha \beta}=R_{\beta \alpha}, \quad B_{\alpha \beta}=-B_{\beta \alpha},
$$

the following rules are also used:

$$
B_{\alpha \beta} R^{\alpha \beta}=0, \quad R_{\alpha \beta} \theta^{\alpha} \theta^{\beta}=0 .
$$

The properties, or the explicit values of components of constant tensors: $\sigma$ matrices, $\eta$ 's and $\epsilon$ 's, have also to be taken into account, but a discussion about it is deferred to the following sections. 


\section{The Algorithm}

There are two major parts, or two major problems, which need to be solved in developing an algorithm and the software for SUSY calculations based on a symbolic programming system. These parts and the algorithms are explained in this section, while the details of the implementation are given in the next one. The various objects (superfields, component fields, constants) have to be defined as well as the actions of operators (covariant, ordinary and Grassman derivatives) on the (super)fields, depending both on the properties of an operator and the index structure of the fields. The other problem is how to take care of the symmetries and how to identify the same terms in an expression; by same we mean the terms that could differ at most by a multiplicative constant.

The first part is a matter of specifying various definitions and dependencies. It does not require any special algorithm, but it is model dependent, so a different set of definitions has to be written for each model. The only point in this part that requires an explanation is the decomposition of a product of two $\sigma$ matrices into terms with definite symmetries, as shown in Example 2 of this section. The definitions needed for $4-D, N=1$ SUSY are given in this package, while, if one wants to work in $2-D, N=2$ SUSY for example, a different set has to be given. The actual implementation of this part, functions that perform the operations and the definitions are explained in Section 4.

On the contrary to the first problem, the second is almost model independent, but requires an algorithm to solve it. The rest of the section is devoted to the algorithm, but before that, something has to be said about the form of an expression that is manipulated; specifically about two different ways the dummy indices could be handled. 
Since Dill is intended to help a physicist by filling some steps in a calculation, it has to accept an expression given in the usual, compact, form as input and to give a modified expression in the same format, as output. 'Usual' means the way a physicist doing such a calculation would put it, which implies the use of the summation convention. The problem is how to handle the dummy (summation) indices. There seem to be two different approaches: either all the manipulations are done on an expression in the compact form, with the dummy indices being symbols (letters), or all the implicit sums in the input expression are first expanded, so that all the dummy indices, given as symbols, are written explicitly (as numbers) then the required manipulation is done, and at the end the appropriate terms are grouped together using the summation convention and dummy indices again. The first, compact method, requires more complicated procedures for the actual manipulations, such as differentiations or symmetry manipulations and can not use the fact that some tensors, ( $\sigma$ matrices, for example), are given constants, while the second, explicit method, needs a complicated procedure that would pull all the terms with explicit indices together, at the end of a calculation. To make this important point clear, let's look at a few examples.

Example 1: Let $R_{\alpha \beta}$ be a symmetric tensor, so:

$$
R_{\alpha \beta} \theta^{\alpha} \theta^{\beta}=0
$$

The explicit method would proceed as follows $(4-D, N=1)$ :

- expansion:

$$
R_{\alpha \beta} \theta^{\alpha} \theta^{\beta}=R_{11} \theta^{1} \theta^{1}+R_{12} \theta^{1} \theta^{2}+R_{21} \theta^{2} \theta^{1}+R_{22} \theta^{2} \theta^{2}
$$

- anticommutation rules:

$$
=R_{12} \theta^{1} \theta^{2}-R_{21} \theta^{1} \theta^{2}
$$


- symmetry of R:

$$
=R_{12} \theta^{1} \theta^{2}-R_{12} \theta^{1} \theta^{2}=0
$$

The compact method requires a much more complicated algorithm (which will be given later) which basically finds that:

$$
\begin{aligned}
R_{\alpha \beta} \theta^{\alpha} \theta^{\beta} & =R_{\beta \alpha} \theta^{\beta} \theta^{\alpha} \\
& =-R_{\alpha \beta} \theta^{\alpha} \theta^{\beta},
\end{aligned}
$$

so that:

$$
R_{\alpha, \beta} \theta^{\alpha} \theta^{\beta}=0
$$

Example 2: Simplification of the expression:

$$
\theta^{\alpha} \sigma_{\alpha \dot{\alpha}}^{n} \bar{\theta}^{\dot{\alpha}} \theta^{\beta} \sigma_{\beta \dot{\beta}}^{k} \bar{\theta}^{\dot{\beta}}
$$

The explicit method would begin with an expansion of the left hand side and explicit values of $k$ and $n$; say $k=1, n=3$, so the previous expression equals:

$$
\begin{aligned}
& =\theta^{1} \sigma^{3}{ }_{1 \dot{1}} \bar{\theta}^{\mathrm{i}} \theta^{1} \sigma^{1}{ }_{1 \mathrm{i}} \bar{\theta}^{\mathrm{i}}+\theta^{1} \sigma^{3}{ }_{1 \dot{1}} \bar{\theta}^{\dot{1}} \theta^{1} \sigma^{1}{ }_{1 \dot{2}} \bar{\theta}^{\dot{2}}+\theta^{1} \sigma^{3}{ }_{1 \dot{1}} \bar{\theta}^{\mathrm{i}} \theta^{2} \sigma^{1}{ }_{2 \dot{1}} \bar{\theta}^{\mathrm{i}} \\
& +\theta^{1} \sigma_{1 \dot{1}}^{3} \bar{\theta}^{\dot{1}} \theta^{2} \sigma_{2 \dot{2}}^{1} \bar{\theta}^{\dot{2}}+\theta^{1} \sigma^{3}{ }_{1 \dot{2}} \bar{\theta}^{\dot{2}} \theta^{1} \sigma^{1}{ }_{1 \dot{1}} \bar{\theta}^{\dot{1}}+\theta^{1} \sigma^{3}{ }_{1 \dot{2}} \bar{\theta}^{\dot{2}} \theta^{1} \sigma^{1}{ }_{1 \dot{2}} \bar{\theta}^{\dot{2}}+\theta^{1} \sigma^{3}{ }_{1 \dot{2}} \bar{\theta}^{\dot{2}} \theta^{2} \sigma_{2}{ }_{2 \dot{1}} \bar{\theta}^{\dot{1}} \\
& +\theta^{1} \sigma_{1 \dot{2}}^{3} \bar{\theta}^{\dot{2}} \theta^{2} \sigma_{2 \dot{2}}^{4} \bar{\theta}^{\dot{2}}+\theta^{2} \sigma_{2 \dot{1}}^{3} \bar{\theta}^{\dot{1}} \theta^{1} \sigma_{1 \dot{1}}^{1} \bar{\theta}^{\dot{1}}+\theta^{2} \sigma_{2 \dot{1}}^{3} \bar{\theta}^{\dot{1}} \theta^{1} \sigma_{1 \dot{2}}^{1} \bar{\theta}^{\dot{2}} \\
& +\theta^{2} \sigma_{2 \dot{1}}^{3} \bar{\theta}^{\mathrm{i}} \theta^{2} \sigma_{2 \dot{1}}^{1} \bar{\theta}^{\mathrm{i}}+\theta^{2} \sigma_{2 \dot{1}}^{3} \bar{\theta}^{\mathrm{i}} \theta^{2} \sigma_{2 \dot{2}}^{5} \bar{\theta}^{\dot{2}}+\theta^{2} \sigma^{3}{ }_{2 \dot{2}} \bar{\theta}^{\dot{2}} \theta^{1} \sigma^{1}{ }_{1 \dot{1}} \bar{\theta}^{\mathrm{i}} \\
& +\theta^{2} \sigma_{2 \dot{2}}^{3} \bar{\theta}^{\dot{2}} \theta^{1} \sigma_{1 \dot{2}}^{1} \bar{\theta}^{\dot{2}}+\theta^{2} \sigma_{2 \dot{2}}^{3} \bar{\theta}^{\dot{2}} \theta^{2} \sigma_{2 \dot{1}}^{1} \bar{\theta}^{\dot{1}}+\theta^{2} \sigma_{2 \dot{2}}^{3} \bar{\theta}^{\dot{2}} \theta^{2} \sigma_{2 \dot{2}}^{6} \bar{\theta}^{\dot{2}} .
\end{aligned}
$$

Using the definition of $\sigma$ matrices:

$$
=\theta^{1} \bar{\theta}^{\dot{1}} \theta^{1} \bar{\theta}^{\dot{2}}+\theta^{1} \bar{\theta}^{\dot{1}} \theta^{2} \bar{\theta}^{\dot{1}}+\left(-\theta^{2} \bar{\theta}^{\dot{2}}\right) \theta^{1} \bar{\theta}^{\dot{2}}+\left(-\theta^{2} \bar{\theta}^{\dot{2}}\right) \theta^{2} \bar{\theta}^{\dot{1}}
$$


anticommutation rules:

$$
=\theta^{1} \theta^{1} \bar{\theta}^{\dot{\mathrm{i}}} \bar{\theta}^{\dot{2}}+\theta^{1} \bar{\theta}^{\dot{\mathrm{i}}} \bar{\theta}^{\dot{\mathrm{i}}} \theta^{2}-\theta^{1} \theta^{2} \bar{\theta}^{\dot{2}} \bar{\theta}^{\dot{2}}-\bar{\theta}^{\dot{\mathrm{i}}} \theta^{2} \theta^{2} \bar{\theta}^{\dot{2}}=0
$$

The whole procedure has to be repeated for all other values of $n$ and $k$.

Since the dummy indices are never written explicitly, the compact method can not use the explicit form of the $\sigma$ matrices. Instead, it looks at the product of two $\sigma$ matrices and decomposes it into a sum of terms with definite symmetries:

$$
\sigma_{\alpha \dot{\alpha}}^{n} \sigma_{\beta \dot{\beta}}^{k}=\sigma_{+++}{ }_{\alpha \beta \dot{\alpha} \dot{\beta}}^{n, k}+\sigma_{+--}{ }_{\alpha \beta \dot{\alpha} \dot{\beta}}^{n, k}+\sigma_{-+-}{ }_{\alpha \beta \dot{\alpha} \dot{\beta}}^{n, k}+\sigma_{--+}{ }_{\alpha \beta \dot{\alpha} \dot{\beta}}^{n, k},
$$

where the first three subscripts denote symmetry $(+)$ or antisymmetry $(-)$ with respect to the interchange of the space-time $(n, k)$, Grassman $(\alpha, \beta)$ and dotted Grassman $(\dot{\alpha}, \dot{\beta})$ indices, respectively. The other combinations do not appear because of the properties of the $\sigma$ matrices. The following identity:

$$
\sigma_{+--}^{n, k}{ }_{\alpha \beta \dot{\alpha} \dot{\beta}}^{n,}=\frac{1}{2} \eta^{n k} \epsilon_{\alpha \beta} \epsilon_{\dot{\alpha} \dot{\beta}}
$$

which comes from the definition of $\sigma_{+--}^{n, k}{ }_{\alpha \beta \dot{\alpha} \dot{\beta}}^{n}$ will be used in a rule later on.

Actually, this is an example of Fierz identities for $4-D, N=1$. They are different for other models, but the logic is the same. Therefore, using Eq.[25], Eq. 21] becomes:

$$
=\sigma_{+++}{ }_{\alpha \beta \dot{\alpha} \dot{\beta}}^{n, k} \theta^{\alpha} \bar{\theta}^{\dot{\alpha}} \theta^{\beta} \bar{\theta}^{\dot{\beta}}+\sigma_{+--}{ }_{\alpha \beta \dot{\alpha} \dot{\beta}}^{n, k} \theta^{\alpha} \bar{\theta}^{\dot{\alpha}} \theta^{\beta} \bar{\theta}^{\dot{\beta}}+\sigma_{-+-}{ }_{\alpha \beta \dot{\alpha} \dot{\beta}}^{n, k} \theta^{\alpha} \bar{\theta}^{\dot{\alpha}} \theta^{\beta} \bar{\theta}^{\dot{\beta}}+\sigma_{--+}{ }_{\alpha \beta \dot{\alpha} \dot{\beta}}^{n, k} \theta^{\alpha} \bar{\theta}^{\dot{\alpha}} \theta^{\beta} \bar{\theta}^{\dot{\beta}}
$$

The symmetry manipulations (explained later on) give:

$$
=\sigma_{+--}{ }_{\alpha \beta \dot{\alpha} \dot{\beta}}^{n, k} \theta^{\alpha} \bar{\theta}^{\dot{\alpha}} \theta^{\beta} \bar{\theta}^{\dot{\beta}} .
$$


Using the previously given rule:

$$
=-\frac{1}{2} \eta^{n k} \epsilon_{\alpha \beta} \epsilon_{\dot{\alpha} \dot{\beta}} \theta^{\alpha} \bar{\theta}^{\dot{\alpha}} \theta^{\beta} \bar{\theta}^{\dot{\beta}}
$$

It is clear, from the previous examples, that the explicit method is more model dependent than the compact one because the number of terms obtained in the expansion depends on the actual dimensions of space-time and $\mathrm{N}$, while the properties of the $\sigma$ matrices, which are model dependent, needed to be defined for both the methods. As was already pointed out, the procedures for the compact method are more complicated, but the problem in the explicit method is how to group terms together once a calculation is done. The method chosen here is the compact one; not only for its apparent beauty - expressions are manipulated in a more human-like way, without expanding the implicit sums, but also because it seems to be easier to generalize for application to other models. In the rest of the section the compact method is presented.

The expression should be in the form of a sum of terms, each term being a product of different factors (fields, constants, ...). Then, the idea is to transform each term so that the factors and the indices are in a previously specified, canonical, form. A term in the canonical form is mathematically the same as the original term; it may only look different because, in general, the factors appear in a different order and the dummy indices are different. A canonical form should be uniquely specified and any two terms that are the same, but have a different form, have to have the same canonical form. Therefore, once all the terms in an expression are brought to the canonical form, the terms that are the same (apart from a multiplicative constant) also look the same, so that the underlying symbolic system can group or cancel them. 
Let us define a few terms that will help understand the definition of the canonical form. All symbolic indices appearing in a given term are classified into two categories: free and dummy indices. Free indices are those that appear only once, while dummy ones appear twice, so that the summation convention applies. Also, all objects that depend on coordinates or have one or more indices are called fields. Commuting numerical factors and constants without indices are called constants.

First, the canonical form is defined and then the transformations needed to bring a term into the order are presented. There are three aspects to be considered for the canonical form: order of the factors, order of dummy indices and symmetry considerations.

\section{The requirements for the canonical form:}

1) Factors are ordered so that constants come first followed by fields sorted according to a user defined list of fields.

2) Symbols for the dummy indices are taken from the beginning of the list of available indices (list of indices without free ones), as many as needed.

3) If a word is constructed by taking all the indices from a term in the order of their appearance, the word that comes from the term in the canonical form appears before all others in dictionary order, where the list of all indices corresponds to an alphabet.

4) A factor that has a symmetry with respect to an index permutation has the indices ordered in the best way, according to the criterion given in the previous requirement.

5) If a term equals zero due to an antisymmetry with respect to an index permutation, the canonical form is simply zero.

It is obvious that the canonical form, defined by the requirements given above, exists 


\begin{tabular}{|c|c|c|}
\hline canonical form & non-canonical form & unsatisfied requirement \\
\hline$a \theta^{\alpha}$ & $\theta^{\alpha} a$ & 1 \\
$\theta^{\gamma} \psi^{\beta}$ & $-\psi^{\beta} \theta^{\gamma}$ & 1 \\
$\theta^{\alpha} \theta^{\beta} \psi_{\alpha}$ & $\theta^{\gamma} \theta^{\beta} \psi_{\gamma}$ & 2 \\
$-\theta^{\beta} \theta^{\gamma}$ & $\theta^{\gamma} \theta^{\beta}$ & 3 \\
$-\theta^{\alpha} \theta_{\alpha} \theta^{\beta} \psi_{\beta}$ & $\theta^{\beta} \theta^{\alpha} \theta_{\alpha} \psi_{\beta}$ & 3 \\
$B_{\beta \gamma}$ & $-B_{\gamma \beta}$ & 4 \\
$-\theta^{\alpha} \theta^{\beta} B_{\alpha \beta}$ & $\theta^{\alpha} \theta^{\beta} B_{\beta \alpha}$ & 4 \\
0 & $\theta^{\alpha} \theta^{\beta} R_{\alpha \beta}$ & 5 \\
\hline
\end{tabular}

Table 1: Canonical ordering

and is unique; the first two requirements can always be satisfied, possibly in more than one way, and the third and the fourth can be also always satisfied; furthermore, they specify the canonical order uniquely due to the properties of the dictionary order.

In order to clarify the definition, examples of canonical forms, together with mathematically same terms not in the canonical form and the number of the canonical form requirement that is not satisfied are given in Table 1. $B_{\alpha \beta}$ is antisymmetric, $R_{\alpha \beta}$ is symmetric with respect to the interchange of indices, and the user defined lists needed are:

list of fields: $\{\theta, \psi, B, R\} \quad$ list of indices: $\{\alpha, \beta, \gamma, \delta\}$.

Also, the upper indices come before the lower ones.

In all the examples given in Table 1 only Grassman indices appear. If more than one type of index is needed, as is usually the case, the canonical form definition essentially stays the same; all the orderings are done for all index types. A list of indices has to be defined 
for each type, so that the dummy indices reassignment is done separately for each index type. The convention used here is that (dotted) Greek letters denote (dotted) Grassman indices and small Latin letters are used for space-time coordinates. Although it would be mathematically correct, it would be odd to use for an index one kind of letter, when another kind is expected.

The main steps in the procedure (algorithm) that transforms a term into the canonical form are first listed and the explanations given immediately after.

\section{Canonical ordering algorithm:}

1) Factors in a term are ordered so that constants come first, followed by fields ordered according to the list of fields.

2) A set of terms is generated by making all the possible permutations of fields within each group of the same fields.

3) Another set of terms is generated from the previous one by using all existing symmetries with respect to index interchange of a field.

4) The dummy indices are reassigned from a list of available indices, according to the order in the list, for every term in the set.

5) A term from the set that has indices ordered in the best way is chosen for the canonical form, unless it is zero due to the symmetries, in which case zero is returned.

In the first step, factors are ordered so that all commutative constants come at the beginning, followed by fields and other anticommuting objects in a user specified order (a user creates a list of fields). Therefore, following constants, groups of one or more of the same fields are created. When this is done, the orders of the anticommuting factors of the 
initial and final factors are compared, so that the parity of a permutation needed to bring one into another is determined and if it is odd, an additional minus sign is introduced in the final term. In this way the minus signs coming from the anticommutation relations are taken into account properly.

There are two types of symmetries that are of concern here: symmetry with respect to a permutation of indices of a single field ( $B_{\alpha \beta}=-B_{\beta \alpha}$, or $T_{\alpha \beta \gamma}=T_{\beta \gamma \alpha}=T_{\gamma \alpha \beta}$ ) and with respect to interchange of the same fields $\left(\theta^{\alpha} \theta^{\beta}=-\theta^{\beta} \theta^{\alpha}\right)$. They both amount to an index permutation which makes them important in finding a canonical form. The term obtained from the previous step is used to generate the terms with all possible permutations of indices that are equal to the term, modulo minus sign. The way it is done is to take each group of the same fields separately, make all possible field permutations within the groups and then combine all the permutations, followed by the usual addition of a minus sign if necessary. Now, the same thing is done with fields that have a definite symmetry with respect to an index permutation (the fields and the corresponding symmetries are specified by the user). The overall sign change depends on the number of antisymmetries used to generate the particular term. What is obtained in this way is a set of terms with all possible index combinations, each equal to the original term.

In the next step of the algorithm, each term from a set is treated separately, so the result will be another set of terms, again each term from the set being equal to the original one. The free indices in a term have to stay as they are; on the other hand, a dummy index can be given a different symbol, provided both of the same indices are given the same, new, symbol. A list of available indices is obtained by removing the free indices of the actual term, from the list of all indices. The dummy indices are reassigned in the order they appear 
in the term: no matter what the actual symbol is, they are assigned a new one, in the order of the available list of indices.

A set of terms obtained in general contains terms that look identical. In order to make further manipulations faster, only distinct terms are retained in the set. If there is more than one kind of index, the reassignment is done with each kind separately, one after another.

What is achieved by the above procedure is that starting from an initial term, a set of terms are generated equal to it. In every term all the fields are in the same order. The terms differ in positions of dummy indices; the symbols used for the dummy indices are the same, but they are assigned in all the possible ways following all the symmetries with respect to permutations of indices and terms.

The final step is to find a term in the canonical form in the set. The first two canonical form requirements are satisfied by construction. As for the next two requirements, a word of indices in the order of their appearance is constructed for every term, all of them are sorted as in a dictionary following the list of all indices and the first one corresponds to a term in the canonical form. If the chosen word appears more than once, the terms corresponding to them can either be identical, in which case it does not matter, or they can differ by an overall minus sign, meaning that the terms equals minus the same term, so it is zero. In this case, in the last step of the algorithm, the term is set to zero. Since all possible symmetries of a term are taken into account for generating the set, if the term is zero mathematically, the same terms with different signs will necessarily appear in the set and will be set to zero. Two examples of transforming expressions to the canonical form, following the steps from the canonical ordering algorithm are given in Appendices A and B.

It has thus been shown that not only does the canonical form of a term (as given by the 
requirements) exist and that it is unique, but also that the procedure given above necessarily leads to it.

\section{Implementation}

The previous discussion was mostly concerned with the algorithm and therefore independent of the actual symbolic programming system used, although the common characteristics of the existing systems were taken into account. In that way, the algorithm presented above can be implemented in anyone of them. This section is in turn devoted to the actual implementation of the algorithm in Mathematica. The functions corresponding to specific manipulations have been written in the Mathematica programming language and the use and the function of the several most important of them is given. The actual form of the functions was strongly influenced by the existing Mathematica commands. If the software is to be written in another similar language the details would have to be different; nevertheless the basic structure would stay the same.

Following the ideas of object oriented programming, the whole package is written in a highly modular form. Every function performs a single well-defined step, so that it is easy to understand the way a function works and it also gives Dill greater flexibility. All the functions in the package can be roughly divided into the main and other functions. A user needs to be familiar with the main functions only, which make up a small fraction of all the functions. The main functions fall in three categories: output commands, format changes and the functions that perform an actual transformation on an expression. The transformations are quite similar to those a physicist would do in an usual calculation. 
The main functions are listed below. The other functions are typically either general, therefore used everywhere, or closely related and providing a necessary step for a specific main function, but are not used by themselves. Unless a user wants to change a function, there is no need to get familiar with them, so they will not be of concern in this paper.

Format changing functions. Before a list and a short description of the functions is given, it is necessary to give an explanation about the forms in which the expressions actually appear during the calculations. Instead of working with an expression in its usual form - fields and constants that are added and multiplied amongst themselves - it was very convenient to establish a few different formats for an expression. A function that performs a certain transformation requires an input expression in a specific form. First of all, given an expression, all multiplications (both commutative and anticommutative) and derivatives (both ordinary and Grassman) have to be distributed over additions, so that the expression is a sum of a product of factors, where a factor can be a constant, a field, or any derivative of any order of a field. This format is called the ordinary expression format. The function initdis acts on any expression and puts it in the ordinary expression form.

If a term of an ordinary expression (a product of constants, fields and derivatives) is written as a list (set) with the factors as elements, the term is in the list format. The whole expression (sum of terms) is in the lists format if it is a list of terms, each term being in the list format. That is, an expression in the lists form is a list of lists of constants, fields and derivatives. This is achieved by the oex2lists function. Further, indices of most of the fields - those listed in raiselist - have to be up. The function used for this is raise.

The last two formats; full for a single term and fulls for the whole expression are again 
similar: a list of terms in the full format makes an expression in the fulls format. A term in the full form is a list of two lists: the first one has constants for elements. The second list contains fields and derivatives grouped in lists of the same fields, as shown in Example 3. The fields are ordered according to funlist. The function lists2fulls transforms an expression in the lists format to fulls. It is strongly recommended that there is no Grassman derivatives nor Kronecker delta functions in the expression.

\section{Example 3:}

Initial expression:

$$
\theta^{\alpha} \theta_{\alpha} \bar{\theta}^{\dot{\alpha}} \bar{\theta}_{\dot{\alpha}}+\left(\frac{\partial}{\partial \theta^{\alpha}} \theta^{\beta} \theta^{\gamma}\right)\left(\psi^{\alpha} \epsilon_{\beta \gamma}\right)-A \frac{\partial}{\partial x^{n}} \frac{\partial}{\partial x^{k}} \eta^{k n}
$$

ordinary expression:

$$
\theta^{\alpha} \theta_{\alpha} \bar{\theta}^{\dot{\alpha}} \bar{\theta}_{\dot{\alpha}}+\left(\frac{\partial}{\partial \theta^{\alpha}} \theta^{\beta}\right) \theta^{\gamma} \psi^{\alpha} \epsilon_{\beta \gamma}-\theta^{\beta}\left(\frac{\partial}{\partial \theta^{\alpha}} \theta^{\gamma}\right) \psi^{\alpha} \epsilon_{\beta \gamma}+A\left(\frac{\partial}{\partial x^{n}} \frac{\partial}{\partial x^{k}} A\right) \eta^{k n}
$$

lists form:

$$
\begin{array}{r}
\left\{\left\{\theta^{\alpha}, \theta^{\beta}, \epsilon_{\alpha \beta}, \bar{\theta}^{\dot{\alpha}}, \bar{\theta}_{\dot{\alpha}}, \epsilon_{\dot{\alpha} \dot{\beta}}\right\},\left\{\frac{\partial}{\partial \theta^{\alpha}} \theta^{\beta}, \theta^{\gamma}, \psi^{\alpha}, \epsilon_{\beta \gamma}\right\}\right. \\
\left.\left\{-1, \theta^{\beta}, \frac{\partial}{\partial \theta^{\alpha}} \theta^{\gamma}, \psi^{\alpha}, \epsilon_{\beta \gamma}\right\},\left\{A, \frac{\partial}{\partial x^{n}} \frac{\partial}{\partial x^{k}} A, \eta^{k n}\right\}\right\}
\end{array}
$$

fulls form:

$$
\begin{aligned}
& \left\{\left\{\{1\},\left\{\left\{\epsilon_{\alpha \beta}\right\},\left\{\bar{\epsilon}_{\dot{\alpha} \dot{\beta}},\right\},\left\{\theta^{\alpha}, \theta^{\beta}\right\},\left\{\bar{\theta}^{\dot{\alpha}}, \bar{\theta}^{\dot{\beta}}\right\}\right\}\right\},\left\{\{1\},\left\{\left\{\frac{\partial}{\partial \theta^{\alpha}} \theta^{\beta}\right\},\left\{\theta^{\gamma}\right\},\left\{\epsilon_{\beta \gamma}\right\},\left\{\psi^{\alpha}\right\}\right\}\right\},\right. \\
& \left.\left\{\{-1\},\left\{\left\{\frac{\partial}{\partial \theta^{\alpha}} \theta^{\gamma}\right\},\left\{\theta^{\beta}\right\},\left\{\epsilon_{\beta \gamma}\right\},\left\{\psi^{\alpha}\right\}\right\}\right\},\left\{\{1\},\left\{\left\{\frac{\partial}{\partial x^{n}} \frac{\partial}{\partial x^{k}} A\right\},\{A\},\left\{\eta^{k n}\right\}\right\}\right\}\right\}
\end{aligned}
$$

In all the following function descriptions, the argument ind stands for an index type, indtypes for a list of index types, funlist for a list of fields and oddlist for a list of fields that have odd Grassman parity. 
The format changing functions are the following:

- initdis [ ex, oddlist ] distributes derivatives, ordinary and noncommutative multiplications over additions in the expression ex and returns the expression in the ordinary expression format.

- raise [ term, indtypes, raiselis ] takes a term in the list form and raises all the indices of the types indty in the factors appearing in raiselis.

- oex2lists [ oex ] transforms an ordinary form expression oex to the list form.

- lists2oex [ ex ] transforms an expression in the lists form to the ordinary expression form.

- list2oex [ term ] transforms a term in the list form to an expression in the ordinary form.

- lists2fulls [ ex, funlist, oddlist ] transforms an expression in the lists form to the fulls form.

- list2full[ term, funlist, oddlist ] transforms a term in the list form to the full form.

- fulls2lists [ ex ] transforms an expression in the fulls form to the lists form.

- full2list [ term ] transforms a term in the full form to the list form.

- oex2fulls [ oex, funlist, oddlist] transforms an ordinary form expression to the fulls form.

- fulls2oex [ ex ] transforms an expression in the fulls form to the ordinary expression form.

Output commands are not necessary but are very convenient. An expression manip- 
ulation or a format changing function returns an expression in the Mathematica expression form (all of the formats discussed above agree with the Mathematica expression form), so that the output expression can be used as an argument of a Mathematica command. Unfortunately it is not easy for a user to read it. Especially in the case of the interactive use, when a user decides about the next step depending on the actual expression, it seems very important to develop a way to display an expression in the same way it would be written by a physicist. For example, the way Dill is used, the expression:

$$
\theta^{\alpha} \sigma_{\alpha \dot{\alpha}}^{n} \bar{\theta}^{\dot{\alpha}} \theta^{\beta} \sigma_{\beta \dot{\beta}}^{k} \bar{\theta}^{\dot{\beta}}
$$

would be in the Mathematica form:

$$
\begin{aligned}
& \left.\operatorname{theta}[\{\text { alpha, } u\}]^{* *} \operatorname{sigma}[\{\mathrm{n}, \mathrm{u}\},\{\text { alpha, } \mathrm{d}\},\{\text { alphad, } \mathrm{d}\}]^{* *} \text { thetab }[\{\text { alphad, } \mathrm{u}\}]\right]^{* *} \\
& \text { theta }[\{\text { beta, } \mathrm{u}\}]^{* *} \operatorname{sigma}[\{\mathrm{k}, \mathrm{u}\},\{\text { beta, } \mathrm{d}\},\{\text { betad, } \mathrm{d}\}]^{* *} \text { thetab }[\{\text { betad, } \mathrm{u}\}] .
\end{aligned}
$$

There is a way to display subscripts and superscripts in an expression built into Mathematica, but it is not flexible enough to use in this package. In order to allow the presentation of an expression in an user - friendly way and to make it easy to save expression in a file, output commands are developed. A user has to specify whether the output should be displayed in $\mathrm{T}_{\mathrm{E}} \mathrm{X}$ format, or in Mathematica format, should be sent to the standard output or to a file, and, in the case that the standard output (usually a screen) and $\mathrm{T}_{\mathrm{E}} \mathrm{X}$ form is chosen, should the results of different manipulations be displayed one by one, as they are calculated, or all together, when the whole job is done. In any case, the output commands affect only the way a user gets a result; Mathematica itself always gets the output in Mathematica form, and is therefore ready for another manipulation. Further, in a program that does several manipulations, the output commands can be used anywhere in the program 
and, at the time the program is run, the user can specify if all of them are to be used, some, or the last one only.

The output commands are:

- outopen[ outdes ] defines parameters needed for writework and outclose. The argument is a list of four elements: the first can be all, some, or last, for all the second "stdout", or a "filename", the third mat, or tex and the fourth tog, or one (all outputs are put together, or appear one by one). Returns a list of three elements.

- writework [ out, string, ex ] sends the expression ex to the output preceded by a word string enclosed in quotes. The argument out is one of the three elements of the list returned by outopen. writework with the argument out being the third element of the list should be used to print the end-result only, writework with the second element of the list should be called after an important intermediate result is generated (generates a short output) and with the first, after each step in the calculation (used for a long output).

- outclose [ outdes ] does whatever is needed to finish the output processes started by outopen. The arguments are the same as for outopen.

- writex $[$ ex $]$ displays the expression ex on the screen as it would be printed by $\mathrm{T}_{\mathrm{E}} \mathrm{X}$. - mrep is a C program that takes a file with Mathematica expressions (whose name is the first argument) and transforms it into a $\mathrm{T}_{\mathrm{E}} \mathrm{X}$ file (whose name is the second argument), by making pattern substitutions defined in the substitution file provided by the user (whose name is the third argument; wb.sub is the substitution file in the package). A pattern/substitution pair can include wild cards. Additional information about mrep and the format of a substitution file are provided in the file mrep.c and in the other source files.

- ftxdvi is a shell script that performs mrep, $\mathrm{T}_{\mathrm{E} X}$ the result and prints it on a screen 
using the funfn xdvi command. The first argument is the name of the input file and the second is the name of the substitution file. The input file and the files generated in the process; a $\mathrm{T}_{\mathrm{E}} \mathrm{X}$ and a dvi file, have the same names; only the extensions are changed appropriately.

- $f t x$ is a shell script similar to ftxdvi. The only difference is that it does not display the output on a screen.

Expression manipulation functions. These functions perform actual calculations, so they are the core of the package. The functions are:

- dman [ ex ] applies the usual rules for Grassman and ordinary derivatives on the expression ex in the lists format. deplist $[\mathrm{x}]$ is needed to specify fields that depend on the space-time coordinate $\mathrm{x}$.

- metricman[ term, indtypes ] takes a list form term term and simplifies products of metrics (index raising function) with upper and lower indices of the types listed in the list indtypes.

- deltaman [ term, indtypes ] takes care of the Kronecker delta function with indices of the types indtypes in the list form term term.

- toodd [ term, ind, oddlist ] multiplies a full form term term by zero if it contains as many anticommuting factors with indices of the ind type as needed for the term to be zero.

- zero [ ex ] deletes the terms from the fulls form expression ex that have a zero factor. - ssigma [ ex, funlist, oddlist ] decomposes a product of two $\sigma$ matrices into the terms with definite symmetries in the fulls form expression ex. 
- canon[ term, out, funlist, oddlist ] puts a full form term into the canonical order and prints the steps if all was an argument to outopen. The argument out is the whole output of outopen. It has to be noted that canon does not order the factors in the term (step 1 in the canonical order algorithm) because it expects the input (term) in the full form, so it is ordered already.

- work [ ex, outdes, funlist, oddlist ] is a program that applies the functions described above, in a certain order, to the input expression ex. The argument outdes is the argument needed for outopen and outclose. The function is listed and its use is shown in the Appendix D.

Since canon is the most important function in the package - it is an implementation of the algorithm given in Section 3 - the functions explicitly called in canon are listed as follows:

- permfac [ term, oddlist ]: makes all possible permutations of the same fields in a full form term term, assigns a sign as needed due to the anticommutation relations and returns a list of terms with the fields permuted; effectively an expression in the fulls form. (Step 2 in the canonical ordering algorithm.)

- symall[ term, ind ]: makes all the permutations of indices of the type ind, according to the (anti)symmetries given by symtype [ field, ind ] lists, in the full form term term and returns a list of the transformed terms. (Step 3 in the canonical ordering algorithm.)

- shuffle [ term, ind [b] ]: reassigns dummy indices of the type ind in the full form term term in the order of their appearance, according to the list of indices with the free indices excluded. (Step 4 in the canonical ordering algorithm.) 
- firstbyind [ ex, indlist ] takes a list of full form terms ex, all of them mathematically the same and returns the list of the terms with the best alphabetical ordering of the indices, according to the list of indices indlist (there may be more than one term with the same index order). If there is more than one term and the terms have different signs a zero is appended to the constant list, effectively setting the term to zero. (Step 5 in the canonical ordering algorithm.)

Two applications of the functions appearing in canon are shown in Appendices A and B.

Definitions and declarations. Also needed are different definitions and declarations:

- funlist: a list of all fields.

- oddlist: a list of all fields with odd Grassman parity.

- deplist $[\mathrm{x}]$ : a list of all fields that depend on space-time.

The following items need to be defined for all index types:

- raiselist [indextype] a list needed in raise.

- ind [ ] a list of all indices of the type ind. An index has a form of a list where the first element is a symbol for the index and the second is $u$, or d denoting up or down position of the index. Must not have any element in common with a similar list for another index type.

- ind $[\mathrm{d}]$ : a list of all up indices of the type ind.

- ind $[u]$ : a list of all down indices of the type ind.

- ind [b]: a list of all index symbols, without the position, of the type ind.

- ind $[\mathrm{ch}]$ : a function that raises the indices of the type ind.

- ind [e]: a list of the explicit index values (integers) of the type ind. (The only thing 
needed in the current implementation is the length of the list, that is the dimension of the (super)space related to the indices.)

The following lists need to be defined for all field and index type combinations:

- symtype [ field, ind ]: a list of all symmetries of field with respect to a permutation of indices of the type ind. Every element of the list is a list; the first element is: $\{1\}$, or $\{-1\}$ for the symmetry or the antisymmetry, and the second is a list denoting the permutation.

Different objects, superfields or covariant derivatives for example, have to be defined if needed. Care has to be taken if an object contains dummy indices within its definition, as is usually the case, because the dummy indices must not have the same symbol as the other indices appearing in the expression containing the object. All the indices appearing in the expression, the dummy indices of the object excluded, have to be listed first, and then the dummy indices can be assigned some other symbols. It would be possible to do it by hand, or to assign the dummy indices from a special set of index symbols not used elsewhere, but it would be very impractical, especially when an expression contains more than one object of the same kind; the dummy indices in all the objects must be different. In order to achieve this a function wbfields is created. It contains definitions for covariant derivatives, vector and scalar fields for $N=1,4-D$ case:

- wbfields[oex ] substitutes objects defined in the function by the definitions, in an ordinary expression oex, in such a way that all the dummy indices are different. For other definitions, wbfields should be used as a template. The name can be changed, or other definitions can be added in the same way the existing ones appear.

The functions can be applied one by one (interactively), or combined in a program (a 
noninteractive case). In the later case, the functions would be applied one after another in a given order. The function work is an example of the program used in the noninteractive case. The expressions obtained after different steps can be easily shown together, or written in a file. This approach is convenient if the calculation takes a long time, but the order of required manipulations is known in advance. Examples of a simplification of an expression using the functions listed above, are given in Appendix $\mathrm{C}$ for the interactive and in Appendix D nor the noninteractive cases respectively.

Other important remarks. Although, as can be seen from the previous function descriptions, several functions that perform a manipulation have as argument not a whole expression, but a term only, it is easy to apply the same function on a whole expression. An expression in both lists or fulls form is a list of terms in the list or the full form:

$$
\text { expression }=\{\text { term_1, term_2, } \ldots\}
$$

so if function takes a term for an argument:

$$
\text { function }[\text { term, ... ] }
$$

where ... stands for other arguments, the construction:

$$
\text { function }[\#, \ldots] \& / @ \text { expression }
$$

gives

$$
\{\text { function }[\text { term_1, .. ], function }[\text { term_2, .. ] }, \ldots\}
$$

that is, it applies function on each term and puts the results in a list, thereby giving the output expression in the same form as the input one. This is important if another function has to be applied to the output expression.

Another important remark concerns the derivatives. While in Mathematica 


$$
D[f, x] \quad \text { stands for: } \quad \frac{\partial}{\partial x} f,
$$

in this package:

$$
\frac{\partial}{\partial x^{n}} f \quad \text { is written as: } \quad d[x[\{n, u\}], f] .
$$

More detailed instructions are provided in the package; they are integrated in the help utility of Mathematica and can be obtained in the same way as information about any other command.

\section{Conclusion}

An algorithm for doing SUSY calculations at the classical level consists of two major parts. The application of operators, definitions and standard rules, such as Fierz identities and Kronecker delta function properties, belong to the first part. The second part relates to transformation of the terms of an expression to a previously specified canonical form using all the symmetry properties of the fields involved, so that when the algorithm is implemented, the underlying symbolic programming system can identify the terms with the same form and group them together or cancel them. The number of terms in a typical supersymmetry calculation and the complicated structure involving different symmetries and summations over many different indices makes the second part much more important. An algorithm for transforming a term into its canonical form, together with a definition of the canonical

form, was found and presented. The algorithm is model independent and it was shown that the canonical form was unique and that the algorithm necessarily lead to it.

The complete algorithm (both parts), for $4-D, N=1$ SUSY theory, was implemented in the Mathematica programming language. Dill, the software package obtained this way, 
consists of functions that perform different manipulations of the first part, a function that brings a term into the canonical form, as well as other functions needed for the proper functioning of the main functions and output commands. The package was tested on several examples, only a few of which are presented in this article. An initial expression was supplied, the appropriate transformations were applied to it and the final result was obtained. It was found that Dill always gives the correct result. In some cases, as shown in Appendix D, the initial expression was not simplified to the fullest extent, because a rule particular to $4-D, N=1$ model was not supplied. Nevertheless, the result obtained is mathematically correct. Therefore, although the package in its current form does not perform all possible manipulations on an expression, the manipulations that it does perform are done correctly. Dill is intended to be an interactive tool (although it can be also used as an ordinary program) and an open set of functions, if another manipulation is needed a function that does it can simply be added. Different models would require some functions and definitions to be changed, but the overall structure and the canonical ordering function can stay the same. The software was run on Sun SPARC-10 workstations. The CPU time needed was typically somewhat bigger, but of the same order than the time an average physicist would need; 10 min compared to 5 min for example.

There are several things that could be done to improve the package. It is always possible to make it faster; the functions were written with the main motivation of working properly. In the course of developing the package a lot of them were simplified and made faster, but there is still space for additional improvements. Another idea would be to incorporate the explicit method explained in Section 3 and use it together with currently used compact method. That would allow simpler implementations of model dependent rules, such as the 
one needed for the complete simplification in Appendix D.

Furthermore, Dill can be extended to include gauge fields and superfields, making it suitable for calculations in super Yang-Mills theories. Apart from additional declarations and definitions, the extension would require rules related to commutators of the Lie algebra used in the theory.

The package is available for free distribution. For the further information please contact the author. 


\section{Appendix A: Canonical Ordering Procedure, Example 1}

In this appendix, the expression: $\theta^{\gamma} \psi^{\beta} \epsilon_{\delta \gamma} \theta^{\delta}$ is transformed according to the canonical ordering procedure given in Section 3. Functions explicitly appearing in canon (see Section 4), that correspond to the steps in the procedure are shown in the way they would be typed by the user.

Initial expression:

$$
\theta^{\gamma} \psi^{\beta} \epsilon_{\delta \gamma} \theta^{\delta}
$$

Step 1 of the canonical ordering algorithm:

$$
-\theta^{\gamma} \theta^{\delta} \epsilon_{\delta \gamma} \psi^{\beta}
$$

Step 2 of the canonical ordering algorithm:

Corresponding command: ex $=\operatorname{permfac}[\operatorname{exps,}$ oddlist $]$;

$$
\left\{-\theta^{\gamma} \theta^{\delta} \epsilon_{\delta \gamma} \psi^{\beta}, \quad \theta^{\delta} \theta^{\gamma} \epsilon_{\delta \gamma} \psi^{\beta}\right\}
$$

Step 3 of the canonical ordering algorithm:

Corresponding command:

$$
\begin{aligned}
& \mathrm{ex}=\text { Flatten }[\operatorname{symall}[\#, \text { lor }] \& / @ \text { ex, } 1] ; \\
& \mathrm{ex}=\text { Flatten }[\operatorname{symall}[\#, \text { gras }] \& / @ \text { ex, } 1] \\
& \mathrm{ex}=\text { Flatten }[\operatorname{symall}[\#, \text { grasd }] \& / @ \mathrm{ex}, 1] ;
\end{aligned}
$$

$$
\left\{-\theta^{\gamma} \theta^{\delta} \epsilon_{\delta \gamma} \psi^{\beta}, \quad \theta^{\delta} \theta^{\gamma} \epsilon_{\delta \gamma} \psi^{\beta}, \quad \theta^{\gamma} \theta^{\delta} \epsilon_{\gamma \delta} \psi^{\beta}, \quad-\theta^{\delta} \theta^{\gamma} \epsilon_{\gamma \delta} \psi^{\beta}\right\}
$$

Step 4 of the canonical ordering algorithm:

Corresponding command:

ex $=\operatorname{shuffle}[\#, \operatorname{lor}[\mathrm{b}]] \& / @$ ex // Union; 


$$
\begin{aligned}
\text { ex }= & \operatorname{shuffle}[\#, \operatorname{gras}[\mathrm{b}]] \& / @ \text { ex // Union; } \\
\text { ex }= & \operatorname{shuffle}[\#, \operatorname{grasd}[\mathrm{b}]] \& / @ \text { ex // Union; } \\
& \left\{-\theta^{\alpha} \theta^{\gamma} \epsilon_{\gamma \alpha} \psi^{\beta}, \quad \theta^{\alpha} \theta^{\gamma} \epsilon_{\alpha \gamma} \psi^{\beta}, \quad \theta^{\alpha} \theta^{\gamma} \epsilon_{\alpha \gamma} \psi^{\beta}, \quad-\theta^{\alpha} \theta^{\gamma} \epsilon_{\gamma \alpha} \psi^{\beta}\right\}
\end{aligned}
$$

Step 5 of the canonical ordering algorithm:

Corresponding command:

$\operatorname{exps}=$ firstbyind $[\operatorname{ex}, \operatorname{Join}[\operatorname{lor}[], \operatorname{gras}[], \operatorname{grasd}[]]][[1]]$;

$$
\theta^{\alpha} \theta^{\gamma} \epsilon_{\alpha \gamma} \psi^{\beta}
$$

\section{Appendix B: Canonical Ordering Procedure, Example 2}

In this appendix, the canonical ordering procedure is applied to the expression:

$$
R_{\gamma \beta} \theta^{\beta} \theta^{\gamma}
$$

Step 1 of the canonical ordering algorithm:

$$
\theta^{\beta} \theta^{\gamma} R_{\gamma \beta}
$$

Step 2 of the canonical ordering algorithm:

Corresponding command: ex $=\operatorname{permfac}[\operatorname{exps}$, oddlist $]$;

$$
\left\{\theta^{\beta} \theta^{\gamma} R_{\gamma \beta}, \quad-\theta^{\gamma} \theta^{\beta} R_{\gamma \beta}\right\}
$$

Step 3 of the canonical ordering algorithm:

Corresponding command:

$$
\begin{aligned}
& \text { ex }=\text { Flatten }[\operatorname{symall}[\#, \text { lor }] \& / @ \text { ex, } 1] ; \\
& \text { ex }=\text { Flatten }[\operatorname{symall}[\#, \text { gras }] \& / @ \text { ex, } 1] ;
\end{aligned}
$$


$\mathrm{ex}=$ Flatten $[\operatorname{symall}[\#$, grasd $] \& / @ \mathrm{ex}, 1]$

$$
\left\{\theta^{\beta} \theta^{\gamma} R_{\gamma \beta}, \quad \theta^{\beta} \theta^{\gamma} R_{\beta \gamma}, \quad-\theta^{\gamma} \theta^{\beta} R_{\gamma \beta}, \quad-\theta^{\gamma} \theta^{\beta} R_{\beta \gamma}\right\}
$$

Step 4 of the canonical ordering algorithm:

Corresponding command:

$$
\begin{aligned}
& \text { ex }=\operatorname{shuffle}[\#, \operatorname{lor}[\mathrm{b}]] \& / @ \text { ex // Union; } \\
& \text { ex }=\operatorname{shuffle}[\#, \operatorname{gras}[\mathrm{b}]] \& / @ \text { ex // Union; } \\
& \text { ex }=\operatorname{shuffle}[\#, \operatorname{grasd}[\mathrm{b}]] \& / @ \text { ex // Union; }
\end{aligned}
$$

$$
\left\{-\theta^{\alpha} \theta^{\beta} R_{\alpha \beta}, \quad-\theta^{\alpha} \theta^{\beta} R_{\beta \alpha}, \quad \theta^{\alpha} \theta^{\beta} R_{\alpha \beta}, \quad \theta^{\alpha} \theta^{\beta} R_{\beta \alpha}\right\}
$$

Step 5 of the canonical ordering algorithm:

Corresponding command:

$\operatorname{exps}=\operatorname{firstbyind}[\operatorname{ex}, \operatorname{Join}[\operatorname{lor}[], \operatorname{gras}[], \operatorname{grasd}[]]][1]]$;

$$
0 \theta^{\alpha} \theta^{\beta} R_{\alpha \beta}=0
$$

\section{Appendix C: Interactive use of the package}

In this example, the expression:

$$
D_{\gamma}\left(\theta^{\delta} \theta_{\delta} F\right)
$$

is evaluated using the functions from the package. The functions needed are shown, together with the output in the $\mathrm{T}_{\mathrm{E}} \mathrm{X}$ mode. This is an example of the interactive use of the package. The output commands that put an output expression in the $\mathrm{T}_{\mathrm{E}} \mathrm{X}$ mode are neglected.

ex $=\operatorname{DSS}[\{$ gamma, d $\}, \operatorname{theta}[\{$ delta, u $\}] * * \operatorname{theta}[\{$ delta, d $\}] * * \mathrm{~F}[]]$ 


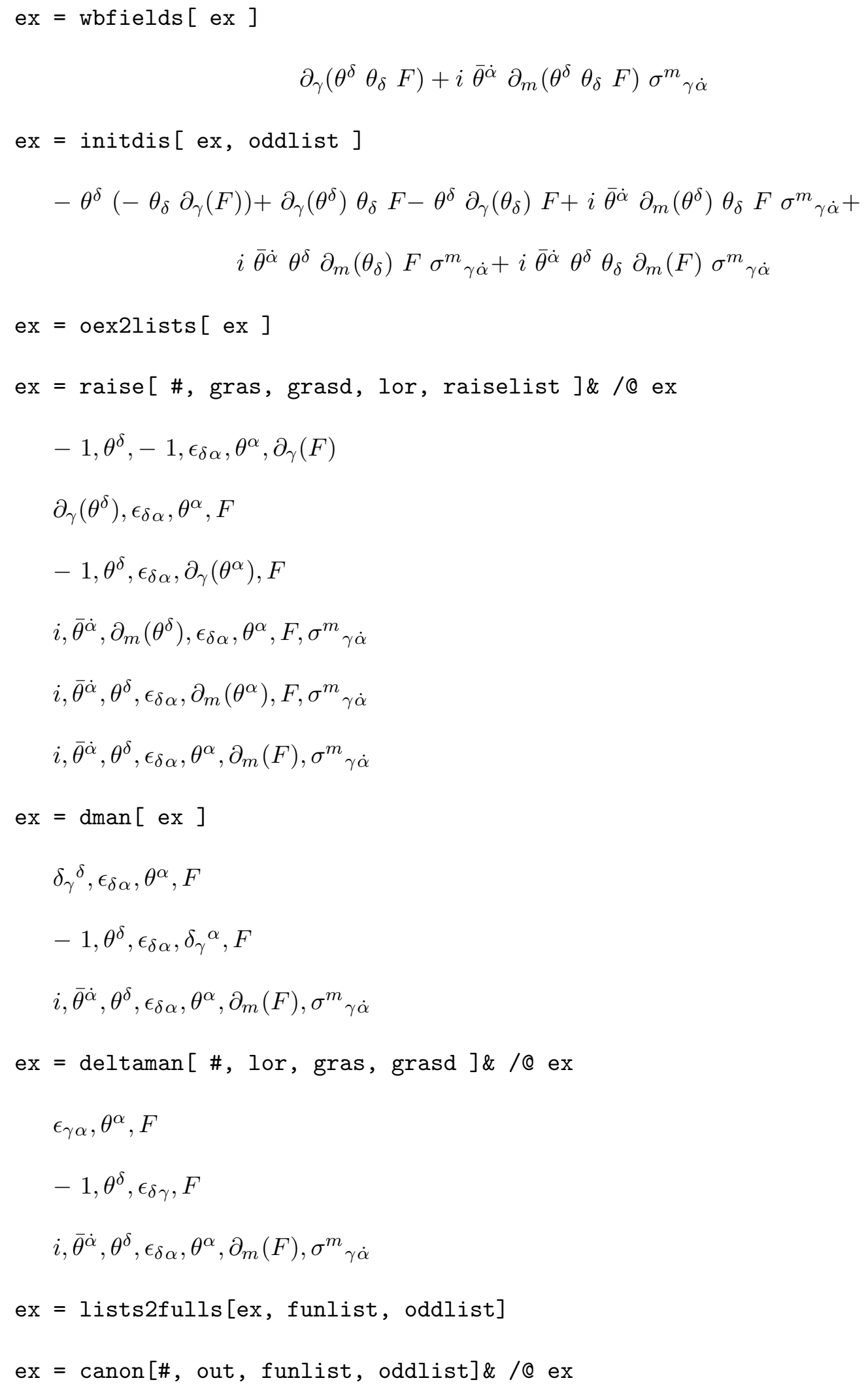




$$
\begin{aligned}
\mathrm{ex} & =\operatorname{zero}[\mathrm{ex}] \\
\mathrm{ex} & =\mathrm{fulls} 20 \mathrm{ex}[\mathrm{ex}] \\
& -2 \theta^{\alpha} \epsilon_{\alpha \gamma} F+i \theta^{\alpha} \theta^{\beta} \bar{\theta}^{\dot{\alpha}} \epsilon_{\alpha \beta} \sigma_{\gamma \dot{\alpha}}^{m} \partial_{m}(F)
\end{aligned}
$$

\section{Appendix D: Noninteractive Use of the Package}

In this example, the expression:

$$
\bar{D}_{\dot{\beta}} \Phi
$$

is evaluated using the program work that contains the functions from the package. The program is shown first, followed by the output. The arguments of the output commands are set to show the output on a screen. The output presented here is exactly the same as the output produced on a screen in the test run. The previously defined lists are the same as in Appendix C.

The program is:

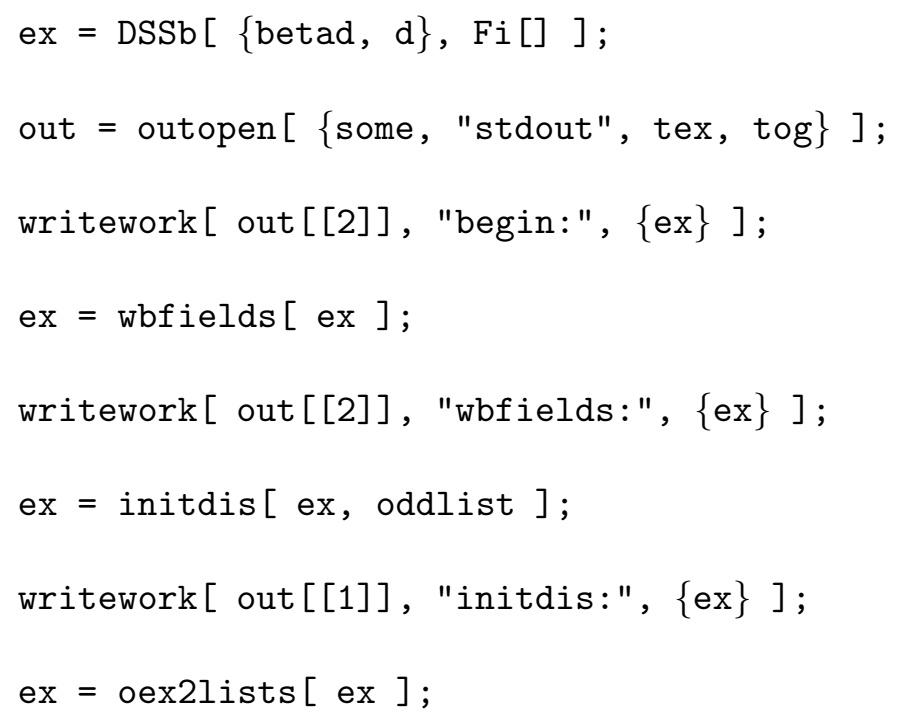




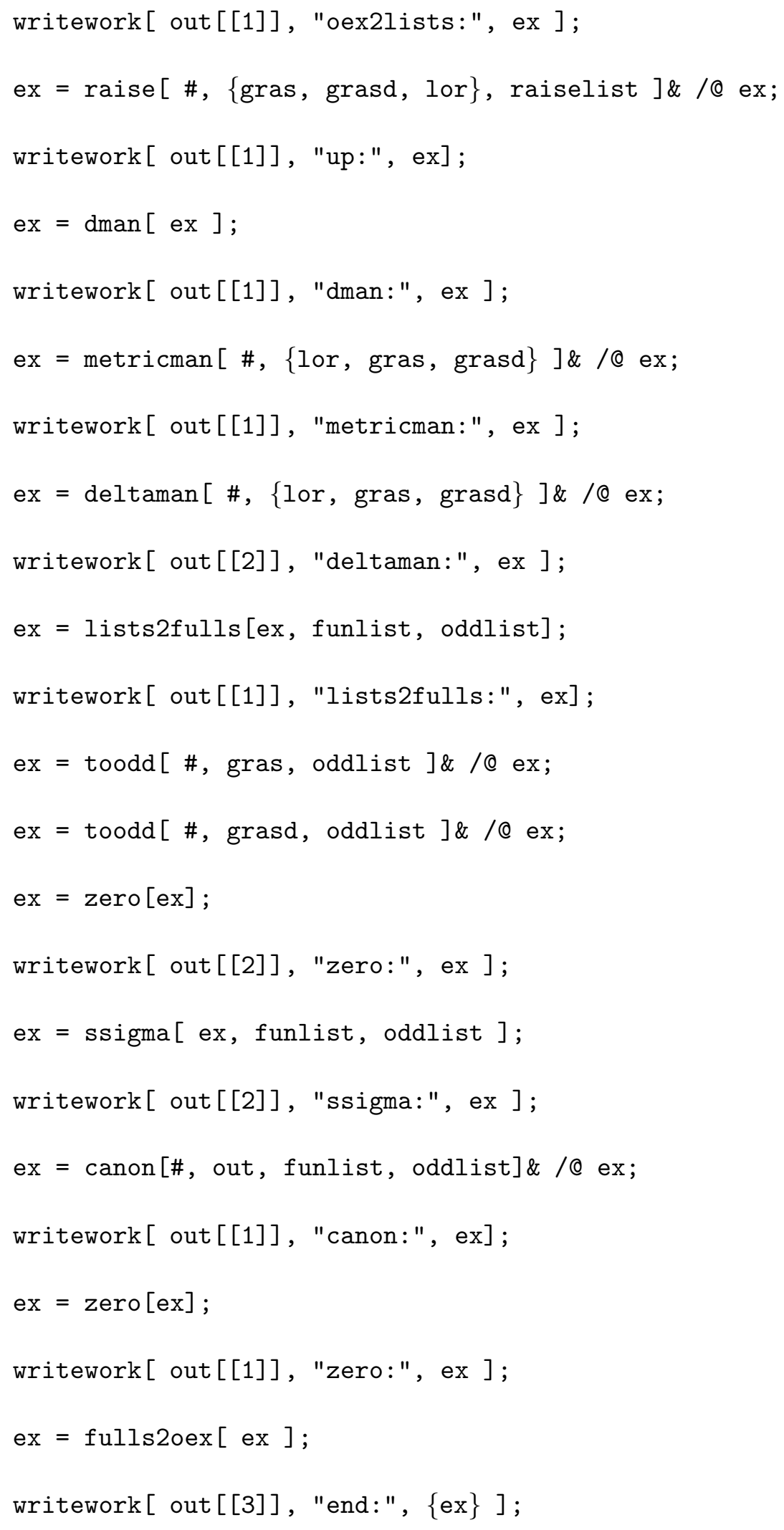


outclose [ some, "stdout", tex, tog\} ];

The output is:

begin:

$\bar{D}_{\dot{\beta}}(\Phi)$

wbfields:

$-\partial_{\dot{\beta}}\left(A+2^{1 / 2} \theta^{\alpha} \psi_{\alpha}+F \theta^{\alpha} \theta_{\alpha}+i \partial_{m}(A) \theta^{\alpha} \sigma_{\alpha \dot{\alpha}}^{m} \bar{\theta}^{\dot{\alpha}}+\left(\partial_{m}\left(\partial_{n}(A)\right) \eta^{m n} \theta^{\alpha} \theta_{\alpha} \bar{\theta}_{\dot{\alpha}} \bar{\theta}^{\dot{\alpha}}\right) / 4-\right.$ $\left.\left(i \theta^{\alpha} \theta_{\alpha} \partial_{m}\left(\psi^{\beta}\right) \bar{\theta}^{\dot{\gamma}} \sigma_{\beta \dot{\gamma}}^{m}\right) / 2^{1 / 2}\right)-i\left(\sigma_{\gamma \dot{\beta}}^{k} \theta^{\gamma}\right) \partial_{k}\left(A+2^{1 / 2} \theta^{\alpha} \psi_{\alpha}+F \theta^{\alpha} \theta_{\alpha}+\right.$

$\left.i \partial_{m}(A) \theta^{\alpha} \sigma_{\alpha \dot{\alpha}}^{m} \bar{\theta}^{\dot{\alpha}}+\left(\partial_{m}\left(\partial_{n}(A)\right) \eta^{m n} \theta^{\alpha} \theta_{\alpha} \bar{\theta}_{\dot{\alpha}} \bar{\theta}^{\dot{\alpha}}\right) / 4-\left(i \theta^{\alpha} \theta_{\alpha} \partial_{m}\left(\psi^{\beta}\right) \bar{\theta}^{\dot{\gamma}} \sigma_{\beta \dot{\gamma}}^{m}\right) / 2^{1 / 2}\right)$

deltaman:

$$
\begin{aligned}
& 1 / 4, \partial_{m}\left(\partial_{n}(A)\right), \eta^{m n}, \theta^{\alpha},-1, \epsilon_{\alpha \beta}, \theta^{\beta},-1, \epsilon_{\dot{\beta} \dot{\gamma}}, \bar{\theta}^{\dot{\gamma}} \\
& 1 / 4, \partial_{m}\left(\partial_{n}(A)\right), \eta^{m n}, \theta^{\alpha},-1, \epsilon_{\alpha \beta}, \theta^{\beta}, \epsilon_{\dot{\alpha} \dot{\beta}}, \bar{\theta}^{\dot{\alpha}} \\
& -i, \sigma_{\gamma \dot{\beta}}^{k}, \theta^{\gamma}, \partial_{k}(A) \\
& -i, \sigma_{\gamma \dot{\beta}}^{k}, \theta^{\gamma}, 2^{1 / 2}, \theta^{\alpha}, \epsilon_{\alpha \beta}, \partial_{k}\left(\psi^{\beta}\right) \\
& -i, \sigma_{\gamma \dot{\beta}}^{k}, \theta^{\gamma}, \partial_{k}(F), \theta^{\alpha}, \epsilon_{\alpha \beta}, \theta^{\beta} \\
& -i, \sigma_{\gamma \dot{\beta}}^{k}, \theta^{\gamma}, i, \partial_{k}\left(\partial_{m}(A)\right), \theta^{\alpha}, \sigma_{\alpha \dot{\alpha}}^{m}, \bar{\theta}^{\dot{\alpha}} \\
& -i, \sigma_{\gamma \dot{\beta}}^{k}, \theta^{\gamma}, 1 / 4, \partial_{k}\left(\partial_{m}\left(\partial_{n}(A)\right)\right), \eta^{m n}, \theta^{\alpha}, \epsilon_{\alpha \beta}, \theta^{\beta}, \epsilon_{\dot{\alpha} \dot{\gamma}}, \bar{\theta}^{\dot{\gamma}}, \bar{\theta}^{\dot{\alpha}}
\end{aligned}
$$

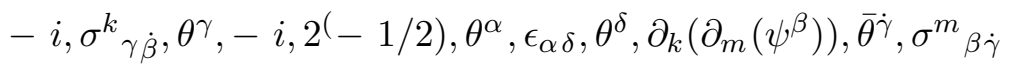

$$
\begin{aligned}
& i, \partial_{m}(A), \theta^{\alpha}, \sigma_{\alpha \dot{\beta}}^{m}
\end{aligned}
$$

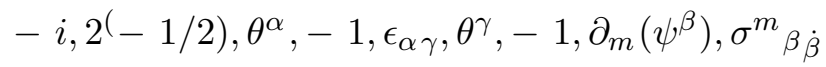

zero:

$$
\begin{aligned}
& 1,1 / 4,-1,-1, \theta^{\alpha}, \theta^{\beta}, \bar{\theta}^{\dot{\gamma}}, \eta^{m n}, \epsilon_{\alpha \beta}, \epsilon_{\dot{\beta} \dot{\gamma}}, \partial_{m}\left(\partial_{n}(A)\right) \\
& 1,1 / 4,-1, \theta^{\alpha}, \theta^{\beta}, \bar{\theta}^{\dot{\alpha}}, \eta^{m n}, \epsilon_{\alpha \beta}, \epsilon_{\dot{\alpha} \dot{\beta}}, \partial_{m}\left(\partial_{n}(A)\right)
\end{aligned}
$$




$$
\begin{aligned}
& 1,-i, \theta^{\gamma}, \sigma^{k}{ }_{\gamma \dot{\beta}}, \partial_{k}(A) \\
& 1,-i, 2^{1 / 2}, \theta^{\gamma}, \theta^{\alpha}, \epsilon_{\alpha \beta}, \sigma^{k}{ }_{\gamma_{\dot{\beta}}}, \partial_{k}\left(\psi^{\beta}\right) \\
& 1,-i, i, \theta^{\gamma}, \theta^{\alpha}, \bar{\theta}^{\dot{\alpha}}, \sigma^{k}{ }_{\gamma_{\dot{\beta}}}, \sigma^{m}{ }_{\alpha \dot{\alpha}}, \partial_{k}\left(\partial_{m}(A)\right) \\
& 1, i, \theta^{\alpha}, \sigma^{m}{ }_{\alpha \dot{\beta}}, \partial_{m}(A) \\
& 1,-i, 2^{(-1 / 2)},-1,-1, \theta^{\alpha}, \theta^{\gamma}, \epsilon_{\alpha \gamma}, \sigma^{m}{ }_{\beta \dot{\beta}}, \partial_{m}\left(\psi^{\beta}\right)
\end{aligned}
$$

ssig:

$$
\begin{aligned}
& 1,1,1 / 4,-1,-1, \theta^{\alpha}, \theta^{\beta}, \bar{\theta}^{\dot{\gamma}}, \eta^{m n}, \epsilon_{\alpha \beta}, \epsilon_{\dot{\beta} \dot{\gamma}}, \partial_{m}\left(\partial_{n}(A)\right) \\
& 1,1,1 / 4,-1, \theta^{\alpha}, \theta^{\beta}, \bar{\theta}^{\dot{\alpha}}, \eta^{m n}, \epsilon_{\alpha \beta}, \epsilon_{\dot{\alpha} \dot{\beta}}, \partial_{m}\left(\partial_{n}(A)\right) \\
& 1,1,-i, \theta^{\gamma}, \sigma_{\gamma \dot{\beta}}^{k}, \partial_{k}(A) \\
& 1,1,-i, 2^{1 / 2}, \theta^{\gamma}, \theta^{\alpha}, \epsilon_{\alpha \beta}, \sigma_{\gamma \dot{\beta}}^{k}, \partial_{k}\left(\psi^{\beta}\right) \\
& 1,1,-i, i, \theta^{\gamma}, \theta^{\alpha}, \bar{\theta}^{\dot{\alpha}}, \sigma_{+++}{ }^{k m}{ }_{\gamma \alpha \dot{\beta}} \dot{\alpha}, \partial_{k}\left(\partial_{m}(A)\right) \\
& 1,1,-i, i, \theta^{\gamma}, \theta^{\alpha}, \bar{\theta}^{\dot{\alpha}}, \sigma_{-+-}{ }^{k m}{ }_{\gamma \alpha \dot{\beta}} \dot{\alpha}, \partial_{k}\left(\partial_{m}(A)\right) \\
& 1,1,-i, i, \theta^{\gamma}, \theta^{\alpha}, \bar{\theta}^{\dot{\alpha}}, \sigma_{--+}{ }^{k m}{ }_{\gamma \alpha \dot{\beta}} \dot{\alpha}, \partial_{k}\left(\partial_{m}(A)\right) \\
& 1,1,-i, i,-1 / 2, \theta^{\gamma}, \theta^{\alpha}, \bar{\theta}^{\dot{\alpha}}, \eta^{k m}, \epsilon_{\gamma \alpha}, \epsilon_{\dot{\beta} \dot{\alpha}}, \partial_{k}\left(\partial_{m}(A)\right) \\
& 1,1, i, \theta^{\alpha}, \sigma^{m}{ }_{\alpha \dot{\beta}}, \partial_{m}(A)
\end{aligned}
$$

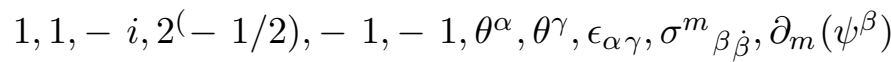

end:

$$
\left(-i \theta^{\alpha} \theta^{\beta} \epsilon_{\alpha \beta} \sigma^{m}{ }_{\gamma \dot{\beta}} \partial_{m}\left(\psi^{\gamma}\right)\right) / 2^{1 / 2}+i 2^{1 / 2} \theta^{\alpha} \theta^{\beta} \epsilon_{\alpha \gamma} \sigma_{\beta \dot{\beta}}^{m} \partial_{m}\left(\psi^{\gamma}\right)
$$

Due to a property of the $4-D, N=1$ model, the last expression equals zero. However, since the package in its current form does not contain a function that would use the property, the initial expression is not simplified to the fullest extent. If such a function is added to the program, the initial expression would be calculated to be zero. 


\section{References}

[1] R. N. Mohapatra, Unification and Supersymmetry (Springer-Verlag, 1992)

[2] L. Castellani, R. D'Auria, P. Fré, Supergravity and Superstrings, a Geometrical Perspective vol. 3 (World Scientific, 1991)

[3] W. H. Klink, Jou. of Comp. Phys. 80 (1989) 453

[4] A. Candiello, Comput. Phys. Commun. 81 (1994) 248, Bulletin board: hep-th@xxx.lanl.gov 9401082

[5] P. Castellvi, E. Lanta and X. Jean, Comput. in Phys. 8 (1994) 360

[6] E. Schrüfer, EXCALC: A System for Doing Calculations in Modern Differential Geometry, User's Manual (Rand Publication, Santa Monica, CA, 1985)

[7] G. C. Joly and M. A. H. MacCallum, Class. and Qu. Grav. 7 (1990) 541

[8] E. Schrúfer, F. W. Hehl, J. D. Mccrea, Gen. Rel. Grav. 19 (1987) 197

[9] M. Jamin, M. E. Lautenbacher, Comput. Phys. Commun 74 (1993) 265

[10] A. Hsieh and E. Yehudai, Comput. in Phys. 6 (1992) 253

[11] T. Kreuzberger, W. Kummer and M. Schweda, Comput. Phys. Commun. 58 (1990) 89

[12] R. Cecchini and M. Tarlini, Comput. Phys. Commun. 60 (1990) 265

[13] U. Hartmann and E. D. Davis, Comput. Phys. Commun. 54 (1989) 353

[14] W. M. Seiler, Comput. Phys. Commun. 66 (1991) 363 
[15] A. C. Hearn, Reduce User's Manual, version 3.2 (Rand Publication CP78, 1985)

[16] L. Castellani, Int. J. Mod. Phys. A vol. 3 no.6 (1988) 1435.

[17] S. Wolfram, Mathematica a System for Doing Mathematics by Computer (AddisonWesley, 1991)

[18] B. Char, O. Goddies, G. Gonnet, B. Leong, M. Monagan and S. Watt, Maple V Language Reference Manual (Springer-Verlag, 1991).

[19] J. Wess and J. Bagger, Supersymmetry and Supergravity (Princeton University Press, 1983).

[20] J. Cornwell, Group Theory in Physics vol. 3 (Academic Press 1990). 